\title{
A Multidimensional Analogue of the Simpson's Formula of Integral
}

\author{
Kazuyuki FUJII * \\ Department of Mathematical Sciences \\ Yokohama City University \\ Yokohama, 236-0027 \\ Japan
}

\begin{abstract}
The Simpson's formula is obtained by approximating the integral of a function on some interval by the integral of the quadratic polynomial determined by the function. However, a multidimensional analogue of the formula has not been given as far as we know.

In this paper such a formula is given. Our formula is simple and beautiful, so it may be convenient in Mathematics or Mathematical Physics.
\end{abstract}

\section{Introduction}

Let $y=f(x)$ be a continuous function defined on the interval $[a, b]$. We want to calculate the integral $\int_{a}^{b} f(x) d x$. However, it is not easy to calculate the integral explicitly, so to make

${ }^{*}$ E-mail address : fujii@yokohama-cu.ac.jp 
some approximation is a realistic way. We recall the Simpson's formula 1 , see for example [1].

We divide $[a, b]$ into equal $2 n$ subintervals and set $\Delta=\frac{b-a}{2 n}$, and $a=x_{0}, b=x_{2 n}$.

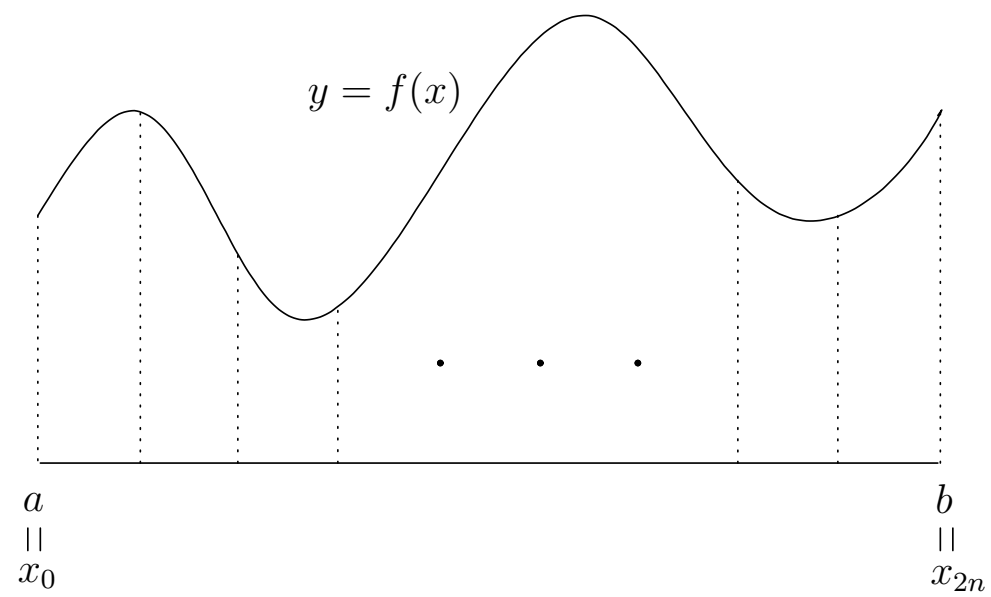

Now we study the integral on small interval $\left[x_{2 k-2}, x_{2 k}\right]$ in the following.

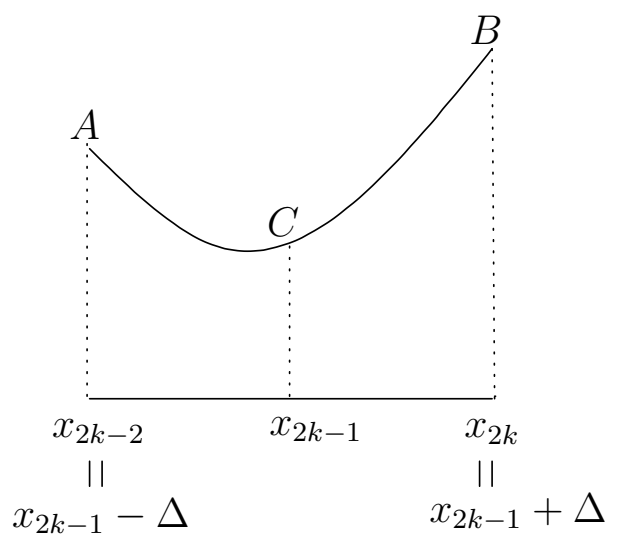

For

$$
y_{2 k-2}=f\left(x_{2 k-2}\right), \quad y_{2 k-1}=f\left(x_{2 k-1}\right), \quad y_{2 k}=f\left(x_{2 k}\right)
$$

we set

$$
\begin{aligned}
A & =\left(x_{2 k-2}, y_{2 k-2}\right)=\left(x_{2 k-1}-\Delta, y_{2 k-2}\right), \\
C & =\left(x_{2 k-1}, y_{2 k-1}\right), \\
B & =\left(x_{2 k}, y_{2 k}\right)=\left(x_{2 k-1}+\Delta, y_{2 k}\right)
\end{aligned}
$$

\footnotetext{
${ }^{1}$ The author uses the terminology Simpson's formula in place of Simpson's rule because it is nothing but an approximate formula (or method)
} 
for simplicity.

A quadratic polynomial

$$
y=p x^{2}+q x+r
$$

passing through three points $A, B, C$ is determined uniquely. Namely, we have only to solve the simultaneous equations

$$
\left\{\begin{array}{l}
\left(x_{2 k-1}-\Delta\right)^{2} p+\left(x_{2 k-1}-\Delta\right) q+r=y_{2 k-2} \\
x_{2 k-1}^{2} p+x_{2 k-1} q+r=y_{2 k-1} \\
\left(x_{2 k-1}+\Delta\right)^{2} p+\left(x_{2 k-1}+\Delta\right) q+r=y_{2 k}
\end{array}\right.
$$

and the result is

$$
\begin{aligned}
p & =\frac{y_{2 k}-2 y_{2 k-1}+y_{2 k-2}}{2 \Delta^{2}} \\
q & =\frac{y_{2 k}-y_{2 k-2}}{2 \Delta}-2 \frac{y_{2 k}-2 y_{2 k-1}+y_{2 k-2}}{2 \Delta^{2}} x_{2 k-1} \\
r & =y_{2 k-1}-\frac{y_{2 k}-y_{2 k-2}}{2 \Delta} x_{2 k-1}+\frac{y_{2 k}-2 y_{2 k-1}+y_{2 k-2}}{2 \Delta^{2}} x_{2 k-1}^{2}
\end{aligned}
$$

Therefore the function $y=f(x)$ on the interval $\left[x_{2 k-2}, x_{2 k}\right]$ is approximated by $y=p x^{2}+$ $q x+r$ like

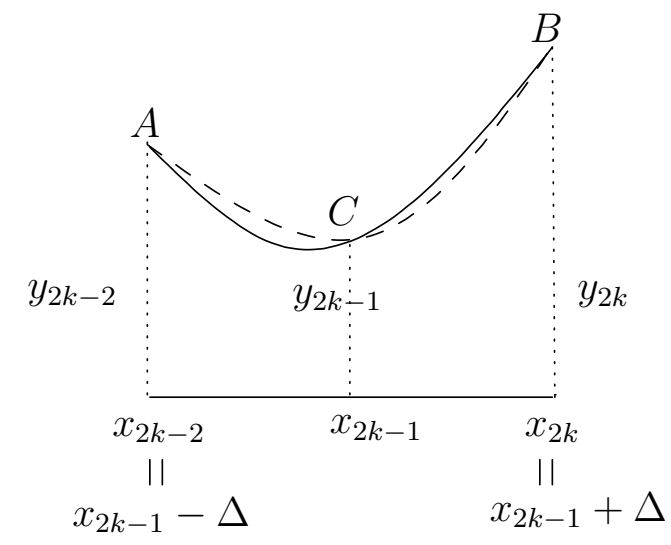

Then an important formula is obtained

$$
\int_{x_{2 k-1}-\Delta}^{x_{2 k-1}+\Delta}\left(p x^{2}+q x+r\right) d x=\frac{\Delta}{3}\left(y_{2 k-2}+4 y_{2 k-1}+y_{2 k}\right)
$$


because

$$
\begin{aligned}
\text { LHS } & =\frac{p}{3}\left\{\left(x_{2 k-1}+\Delta\right)^{3}-\left(x_{2 k-1}-\Delta\right)^{3}\right\}+\frac{q}{2}\left\{\left(x_{2 k-1}+\Delta\right)^{2}-\left(x_{2 k-1}-\Delta\right)^{2}\right\}+2 r \Delta \\
& =\frac{2}{3} p \Delta^{3}+2\left(p x_{2 k-1}^{2}+q x_{2 k-1}+r\right) \Delta \\
& =\frac{2}{3} p \Delta^{3}+2 y_{2 k-1} \Delta \\
& =\frac{\Delta}{3}\left\{2 \Delta^{2} \times \frac{y_{2 k}-2 y_{2 k-1}+y_{2 k-2}}{2 \Delta^{2}}+6 y_{2 k-1}\right\} \\
& =\frac{\Delta}{3}\left(y_{2 k-2}+4 y_{2 k-1}+y_{2 k}\right)=\text { RHS. }
\end{aligned}
$$

From this we have the famous Simpson's formula

$$
\begin{aligned}
\int_{a}^{b} f(x) d x & =\sum_{k=1}^{n} \int_{x_{2 k-2}}^{x_{2 k}} f(x) d x=\sum_{k=1}^{n} \int_{x_{2 k-1}-\Delta}^{x_{2 k-1}+\Delta} f(x) d x \\
& \approx \sum_{k=1}^{n} \int_{x_{2 k-1}-\Delta}^{x_{2 k-1}+\Delta}\left(p x^{2}+q x+r\right) d x \\
& =\sum_{k=1}^{n} \frac{\Delta}{3}\left(y_{2 k-2}+4 y_{2 k-1}+y_{2 k}\right) \\
& =\frac{b-a}{6 n}\left\{y_{0}+4\left(y_{1}+y_{3}+\cdots+y_{2 n-1}\right)+2\left(y_{2}+y_{4}+\cdots+y_{2 n-2}\right)+y_{2 n}\right\}
\end{aligned}
$$

because $\Delta=\frac{b-a}{2 n}$.

\section{Generalized Simpson's Formula}

Let $D$ be an $n$-dimensional cuboid

$$
D=\left[a_{1}, b_{1}\right] \times\left[a_{2}, b_{2}\right] \times \cdots\left[a_{n}, b_{n}\right] \subset \mathbf{R}^{n}
$$

and $f$ be a continuous function on $D$. What we do is to calculate the multidimensional integral

$$
\iint \cdots \int_{D} f\left(x_{1}, x_{2}, \cdots, x_{n}\right) d x_{1} d x_{2} \cdots d x_{n}
$$

However, it is almost difficult, so we must satisfy only by approximating it as shown in the introduction. For that purpose we rewrite $D$ as follows.

$$
D=\left[\alpha_{1}-\Delta_{1}, \alpha_{1}+\Delta_{1}\right] \times\left[\alpha_{2}-\Delta_{2}, \alpha_{2}+\Delta_{2}\right] \times \cdots \times\left[\alpha_{n}-\Delta_{n}, \alpha_{n}+\Delta_{n}\right]
$$


where $\alpha_{j}=\left(b_{j}+a_{j}\right) / 2$ and $\Delta_{j}=\left(b_{j}-a_{j}\right) / 2$. See the following figure $(n=2)$.

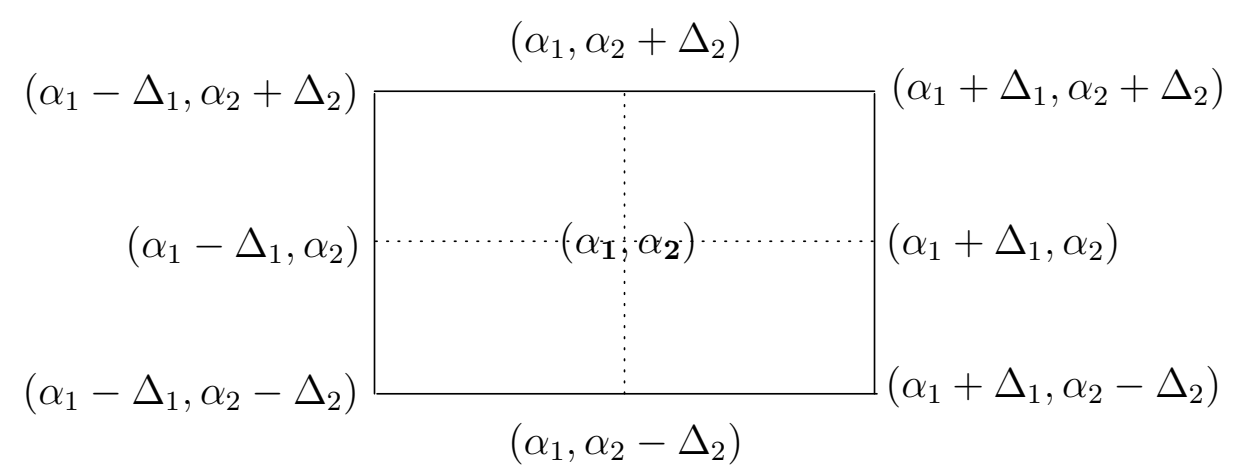

Next we approximate $f$ by a quadratic polynomial

$$
F\left(x_{1}, x_{2}, \cdots, x_{n}\right)=\sum_{i_{1}, i_{2}, \cdots, i_{n}=0}^{2} a_{i_{1} i_{2} \cdots i_{n}} x_{1}^{i_{1}} x_{2}^{i_{2}} \cdots x_{n}^{i_{n}},
$$

which is a natural extension in case of $n=1$.

For $j_{1}, j_{2}, \cdots, j_{n} \in\{-1,0,1\}$ we set

$$
\begin{aligned}
w_{j_{1} j_{2} \cdots j_{n}} & =f\left(\alpha_{1}+j_{1} \Delta_{1}, \alpha_{2}+j_{2} \Delta_{2}, \cdots, \alpha_{n}+j_{n} \Delta_{n}\right) \\
& =F\left(\alpha_{1}+j_{1} \Delta_{1}, \alpha_{2}+j_{2} \Delta_{2}, \cdots, \alpha_{n}+j_{n} \Delta_{n}\right) .
\end{aligned}
$$

It is of course

$$
\sharp\left\{w_{j_{1} j_{2} \cdots j_{n}}\right\}=3^{n}=\sharp\left\{a_{i_{1} i_{2} \cdots i_{n}}\right\},
$$

see the figure above. Then we have

\section{Formula (Conjecture)}

$$
\begin{aligned}
& \iint \cdots \int_{D} F\left(x_{1}, x_{2}, \cdots, x_{n}\right) d x_{1} d x_{2} \cdots d x_{n} \\
& =\frac{\Delta_{1} \Delta_{2} \cdots \Delta_{n}}{3^{n}} \sum_{j_{1}, j_{2}, \cdots, j_{n} \in\{-1,0,1\}} 4^{\sharp\left\{j_{1}, j_{2}, \cdots, j_{n}\right\}_{0}} w_{j_{1} j_{2} \cdots j_{n}}
\end{aligned}
$$

where $\sharp\left\{j_{1}, j_{2}, \cdots, j_{n}\right\}_{0}$ is the number of 0 in $\left\{j_{1}, j_{2}, \cdots, j_{n}\right\}$.

For the case of $n=2$ and $n=3$ the formula is proved in the following. However, the proof of the general case is left to readers. 
From the formula we obtain a good approximation

$$
\begin{aligned}
& \iint \cdots \int_{D} f\left(x_{1}, x_{2}, \cdots, x_{n}\right) d x_{1} d x_{2} \cdots d x_{n} \\
& \approx \frac{\Delta_{1} \Delta_{2} \cdots \Delta_{n}}{3^{n}} \sum_{j_{1}, j_{2}, \cdots, j_{n} \in\{-1,0,1\}} 4^{\sharp\left\{j_{1}, j_{2}, \cdots, j_{n}\right\}_{0}} w_{j_{1} j_{2} \cdots j_{n}}
\end{aligned}
$$

if $D$ is small enough.

\subsection{Proof for $n=2$}

Let us prove (15) for $n=2$. From (3) we set for simplicity

$$
\begin{aligned}
w & =F(x, y)=\sum_{i, j=0}^{2} a_{i j} x^{i} y^{j} \\
& =a_{20} x^{2}+a_{10} x+a_{00}+\left(a_{21} x^{2}+a_{11} x+a_{01}\right) y+\left(a_{22} x^{2}+a_{12} x+a_{02}\right) y^{2} .
\end{aligned}
$$

See the figure once more.

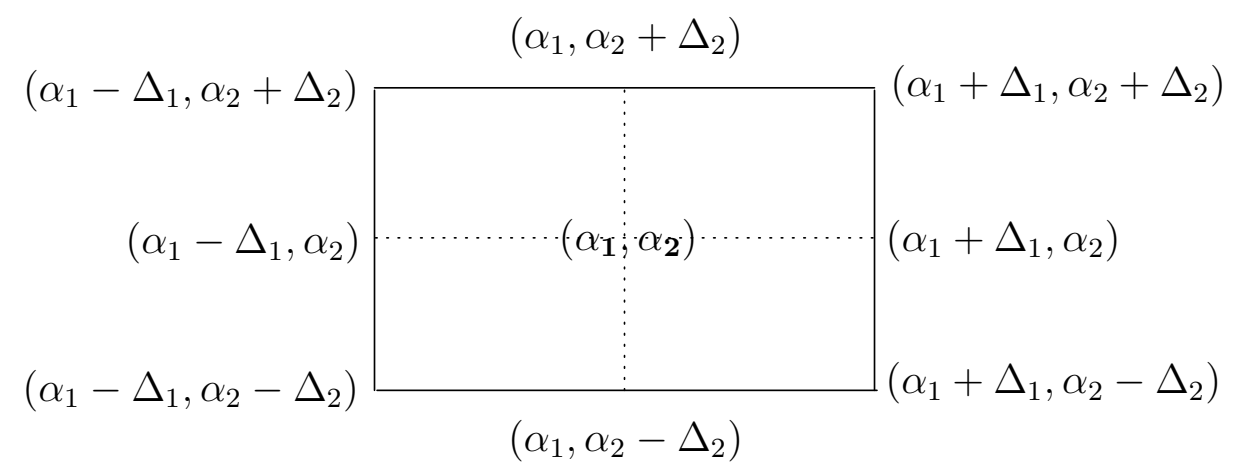


Then

$$
\begin{aligned}
& \int_{\alpha_{1}-\Delta_{1}}^{\alpha_{1}+\Delta_{1}} \int_{\alpha_{2}-\Delta_{2}}^{\alpha_{2}+\Delta_{2}} w d x d y \\
= & \left\{a_{20} \frac{\left(\alpha_{1}+\Delta_{1}\right)^{3}-\left(\alpha_{1}-\Delta_{1}\right)^{3}}{3}+a_{10} \frac{\left(\alpha_{1}+\Delta_{1}\right)^{2}-\left(\alpha_{1}-\Delta_{1}\right)^{2}}{2}+a_{00} \cdot 2 \Delta_{1}\right\} 2 \Delta_{2} \\
+ & \left\{a_{21} \frac{\left(\alpha_{1}+\Delta_{1}\right)^{3}-\left(\alpha_{1}-\Delta_{1}\right)^{3}}{3}+a_{11} \frac{\left(\alpha_{1}+\Delta_{1}\right)^{2}-\left(\alpha_{1}-\Delta_{1}\right)^{2}}{2}+a_{01} \cdot 2 \Delta_{1}\right\} \times \\
& \frac{\left(\alpha_{2}+\Delta_{2}\right)^{2}-\left(\alpha_{2}-\Delta_{2}\right)^{2}}{2} \\
+ & \left\{a_{22} \frac{\left(\alpha_{1}+\Delta_{1}\right)^{3}-\left(\alpha_{1}-\Delta_{1}\right)^{3}}{3}+a_{12} \frac{\left(\alpha_{1}+\Delta_{1}\right)^{2}-\left(\alpha_{1}-\Delta_{1}\right)^{2}}{2}+a_{02} \cdot 2 \Delta_{1}\right\} \times \\
& \frac{\left(\alpha_{2}+\Delta_{2}\right)^{3}-\left(\alpha_{2}-\Delta_{2}\right)^{3}}{3} \\
= & \cdots \\
= & 4 \Delta_{1} \Delta_{2}\left\{a_{20} \alpha_{1}^{2}+a_{10} \alpha_{1}+a_{00}+\left(a_{21} \alpha_{1}^{2}+a_{11} \alpha_{1}+a_{01}\right) \alpha_{2}+\left(a_{22} \alpha_{1}^{2}+a_{12} \alpha_{1}+a_{02}\right) \alpha_{2}^{2}\right\} \\
+ & \frac{4 \Delta_{1} \Delta_{2}}{3}\left(a_{20} \Delta_{1}^{2}+a_{21} \Delta_{1}^{2} \alpha_{2}+a_{22} \Delta_{1}^{2} \alpha_{2}^{2}+a_{02} \Delta_{2}^{2}+a_{12} \alpha_{1} \Delta_{2}^{2}+a_{22} \alpha_{1}^{2} \Delta_{2}^{2}\right) \\
+ & \frac{4 \Delta_{1} \Delta_{2}}{9} a_{22} \Delta_{1}^{2} \Delta_{2}^{2} .
\end{aligned}
$$

From (4) we have $9\left(=3^{2}\right)$-data

$$
w_{k, l}=w\left(\alpha_{1}+k \Delta_{1}, \alpha_{2}+l \Delta_{2}\right)
$$

for $k, l \in\{-1,0,1\}$, so we want to express the integral above in terms of these data $\left\{w_{k l}\right\}$. Since

$$
a_{20} \alpha_{1}^{2}+a_{10} \alpha_{1}+a_{00}+\left(a_{21} \alpha_{1}^{2}+a_{11} \alpha_{1}+a_{01}\right) \alpha_{2}+\left(a_{22} \alpha_{1}^{2}+a_{12} \alpha_{1}+a_{02}\right) \alpha_{2}^{2}=w_{0,0}
$$

is clear we must treat the remaining ones

$$
\begin{aligned}
& a_{20} \Delta_{1}^{2}+a_{21} \Delta_{1}^{2} \alpha_{2}+a_{22} \Delta_{1}^{2} \alpha_{2}^{2}+a_{02} \Delta_{2}^{2}+a_{12} \alpha_{1} \Delta_{2}^{2}+a_{22} \alpha_{1}^{2} \Delta_{2}^{2} \\
& a_{22} \Delta_{1}^{2} \Delta_{2}^{2}
\end{aligned}
$$

It is not difficult to show

$$
\begin{aligned}
a_{20} \Delta_{1}^{2}+a_{21} \Delta_{1}^{2} \alpha_{2}+a_{22} \Delta_{1}^{2} \alpha_{2}^{2} & =\frac{w_{-1,0}-2 w_{0,0}+w_{1,0}}{2} \\
a_{02} \Delta_{2}^{2}+a_{12} \alpha_{1} \Delta_{2}^{2}+a_{22} \alpha_{1}^{2} \Delta_{2}^{2} & =\frac{w_{0,-1}-2 w_{0,0}+w_{0,1}}{2},
\end{aligned}
$$


so we have

$$
\begin{aligned}
& a_{20} \Delta_{1}^{2}+a_{21} \Delta_{1}^{2} \alpha_{2}+a_{22} \Delta_{1}^{2} \alpha_{2}^{2}+a_{02} \Delta_{2}^{2}+a_{12} \alpha_{1} \Delta_{2}^{2}+a_{22} \alpha_{1}^{2} \Delta_{2}^{2} \\
= & \frac{w_{-1,0}+w_{1,0}+w_{0,-1}+w_{0,1}-4 w_{0,0}}{2}
\end{aligned}
$$

Similarly,

$$
\begin{aligned}
& \frac{w_{-1,-1}+w_{-1,1}+w_{1,-1}+w_{1,1}}{4} \\
= & a_{20} \alpha_{1}^{2}+a_{10} \alpha_{1}+a_{00}+\left(a_{21} \alpha_{1}^{2}+a_{11} \alpha_{1}+a_{01}\right) \alpha_{2}+\left(a_{22} \alpha_{1}^{2}+a_{12} \alpha_{1}+a_{02}\right) \alpha_{2}^{2} \\
+ & a_{20} \Delta_{1}^{2}+a_{21} \Delta_{1}^{2} \alpha_{2}+a_{22} \Delta_{1}^{2} \alpha_{2}^{2}+a_{02} \Delta_{2}^{2}+a_{12} \alpha_{1} \Delta_{2}^{2}+a_{22} \alpha_{1}^{2} \Delta_{2}^{2} \\
+ & a_{22} \Delta_{1}^{2} \Delta_{2}^{2} \\
= & w_{0,0}+\frac{w_{-1,0}+w_{1,0}+w_{0,-1}+w_{0,1}-4 w_{0,0}}{2}+a_{22} \Delta_{1}^{2} \Delta_{2}^{2}
\end{aligned}
$$

so we have

$$
a_{22} \Delta_{1}^{2} \Delta_{2}^{2}=\frac{w_{-1,-1}+w_{-1,1}+w_{1,-1}+w_{1,1}-2\left(w_{-1,0}+w_{1,0}+w_{0,-1}+w_{0,1}\right)+4 w_{0,0}}{4}
$$

Therefore, substituting (10), (11) and (12) into (8) we obtain

$$
\begin{aligned}
& \int_{\alpha_{1}-\Delta_{1}}^{\alpha_{1}+\Delta_{1}} \int_{\alpha_{2}-\Delta_{2}}^{\alpha_{2}+\Delta_{2}} w d x d y \\
= & 4 \Delta_{1} \Delta_{2} w_{0,0}+\frac{2 \Delta_{1} \Delta_{2}}{3}\left(w_{-1,0}+w_{1,0}+w_{0,-1}+w_{0,1}-4 w_{0,0}\right) \\
+ & \frac{\Delta_{1} \Delta_{2}}{9}\left(w_{-1,-1}+w_{-1,1}+w_{1,-1}+w_{1,1}-2\left(w_{-1,0}+w_{1,0}+w_{0,-1}+w_{0,1}\right)+4 w_{0,0}\right) \\
= & \frac{\Delta_{1} \Delta_{2}}{9}\left\{w_{-1,-1}+w_{-1,1}+w_{1,-1}+w_{1,1}+4\left(w_{-1,0}+w_{1,0}+w_{0,-1}+w_{0,1}\right)+16 w_{0,0}\right\} .
\end{aligned}
$$

This formula is interesting enough. 


\subsection{Proof for $n=3$}

Let us prove (5) for $n=3$. From (3) we set

$$
\begin{aligned}
w & =F(x, y, z)=\sum_{i, j, k=0}^{2} a_{i j k} x^{i} y^{j} z^{k} \\
& =a_{200} x^{2}+a_{100} x+a_{000}+\left(a_{210} x^{2}+a_{110} x+a_{010}\right) y+\left(a_{220} x^{2}+a_{120} x+a_{020}\right) y^{2} \\
& +\left\{a_{201} x^{2}+a_{101} x+a_{001}+\left(a_{211} x^{2}+a_{111} x+a_{011}\right) y+\left(a_{221} x^{2}+a_{121} x+a_{021}\right) y^{2}\right\} z \\
& +\left\{a_{202} x^{2}+a_{102} x+a_{002}+\left(a_{212} x^{2}+a_{112} x+a_{012}\right) y+\left(a_{222} x^{2}+a_{122} x+a_{022}\right) y^{2}\right\} z^{2}
\end{aligned}
$$

Then

$$
\begin{aligned}
& \int_{\alpha_{1}-\Delta_{1}}^{\alpha_{1}+\Delta_{1}} \int_{\alpha_{2}-\Delta_{2}}^{\alpha_{2}+\Delta_{2}} \int_{\alpha_{3}-\Delta_{3}}^{\alpha_{3}+\Delta_{3}} w d x d y d z \\
= & \left\{\int_{\alpha_{1}-\Delta_{1}}^{\alpha_{1}+\Delta_{1}} \int_{\alpha_{2}-\Delta_{2}}^{\alpha_{2}+\Delta_{2}} \sum_{i, j=0}^{2} a_{i j 0} x^{i} y^{j}\right\} 2 \Delta_{3} \\
+ & \left\{\int_{\alpha_{1}-\Delta_{1}}^{\alpha_{1}+\Delta_{1}} \int_{\alpha_{2}-\Delta_{2}}^{\alpha_{2}+\Delta_{2}} \sum_{i, j=0}^{2} a_{i j 1} x^{i} y^{j}\right\} \frac{\left(\alpha_{3}+\Delta_{3}\right)^{2}-\left(\alpha_{3}-\Delta_{3}\right)^{2}}{2} \\
+ & \left\{\int_{\alpha_{1}-\Delta_{1}}^{\alpha_{1}+\Delta_{1}} \int_{\alpha_{2}-\Delta_{2}}^{\alpha_{2}+\Delta_{2}} \sum_{i, j=0}^{2} a_{i j 2} x^{i} y^{j}\right\} \frac{\left(\alpha_{3}+\Delta_{3}\right)^{3}-\left(\alpha_{3}-\Delta_{3}\right)^{3}}{3} .
\end{aligned}
$$

By (8) some calculation gives 


$$
\begin{aligned}
& \int_{\alpha_{1}-\Delta_{1}}^{\alpha_{1}+\Delta_{1}} \int_{\alpha_{2}-\Delta_{2}}^{\alpha_{2}+\Delta_{2}} \int_{\alpha_{3}-\Delta_{3}}^{\alpha_{3}+\Delta_{3}} w d x d y d z \\
& =\left[4 \Delta_{1} \Delta_{2}\left\{a_{200} \alpha_{1}^{2}+a_{100} \alpha_{1}+a_{000}+\left(a_{210} \alpha_{1}^{2}+a_{110} \alpha_{1}+a_{010}\right) \alpha_{2}+\left(a_{220} \alpha_{1}^{2}+a_{120} \alpha_{1}+a_{020}\right) \alpha_{2}^{2}\right\}\right. \\
& +\frac{4 \Delta_{1} \Delta_{2}}{3}\left(a_{200} \Delta_{1}^{2}+a_{210} \Delta_{1}^{2} \alpha_{2}+a_{220} \Delta_{1}^{2} \alpha_{2}^{2}+a_{020} \Delta_{2}^{2}+a_{120} \alpha_{1} \Delta_{2}^{2}+a_{220} \alpha_{1}^{2} \Delta_{2}^{2}\right) \\
& \left.+\frac{4 \Delta_{1} \Delta_{2}}{9} a_{220} \Delta_{1}^{2} \Delta_{2}^{2}\right] 2 \Delta_{3} \\
& +\left[4 \Delta_{1} \Delta_{2}\left\{a_{201} \alpha_{1}^{2}+a_{101} \alpha_{1}+a_{001}+\left(a_{211} \alpha_{1}^{2}+a_{111} \alpha_{1}+a_{011}\right) \alpha_{2}+\left(a_{221} \alpha_{1}^{2}+a_{121} \alpha_{1}+a_{021}\right) \alpha_{2}^{2}\right\}\right. \\
& +\frac{4 \Delta_{1} \Delta_{2}}{3}\left(a_{201} \Delta_{1}^{2}+a_{211} \Delta_{1}^{2} \alpha_{2}+a_{221} \Delta_{1}^{2} \alpha_{2}^{2}+a_{021} \Delta_{2}^{2}+a_{121} \alpha_{1} \Delta_{2}^{2}+a_{221} \alpha_{1}^{2} \Delta_{2}^{2}\right) \\
& \left.+\frac{4 \Delta_{1} \Delta_{2}}{9} a_{221} \Delta_{1}^{2} \Delta_{2}^{2}\right] 2 \alpha_{3} \Delta_{3} \\
& +\left[4 \Delta_{1} \Delta_{2}\left\{a_{202} \alpha_{1}^{2}+a_{102} \alpha_{1}+a_{002}+\left(a_{212} \alpha_{1}^{2}+a_{112} \alpha_{1}+a_{012}\right) \alpha_{2}+\left(a_{222} \alpha_{1}^{2}+a_{122} \alpha_{1}+a_{022}\right) \alpha_{2}^{2}\right\}\right. \\
& +\frac{4 \Delta_{1} \Delta_{2}}{3}\left(a_{202} \Delta_{1}^{2}+a_{212} \Delta_{1}^{2} \alpha_{2}+a_{222} \Delta_{1}^{2} \alpha_{2}^{2}+a_{022} \Delta_{2}^{2}+a_{122} \alpha_{1} \Delta_{2}^{2}+a_{222} \alpha_{1}^{2} \Delta_{2}^{2}\right) \\
& \left.+\frac{4 \Delta_{1} \Delta_{2}}{9} a_{222} \Delta_{1}^{2} \Delta_{2}^{2}\right]\left(2 \alpha_{3}^{2} \Delta_{3}+\frac{2}{3} \Delta_{3}^{3}\right) \\
& =8 \Delta_{1} \Delta_{2} \Delta_{3} \times \\
& {\left[\left\{a_{200} \alpha_{1}^{2}+a_{100} \alpha_{1}+a_{000}+\left(a_{210} \alpha_{1}^{2}+a_{110} \alpha_{1}+a_{010}\right) \alpha_{2}+\left(a_{220} \alpha_{1}^{2}+a_{120} \alpha_{1}+a_{020}\right) \alpha_{2}^{2}\right\}\right.} \\
& +\left\{a_{201} \alpha_{1}^{2}+a_{101} \alpha_{1}+a_{001}+\left(a_{211} \alpha_{1}^{2}+a_{111} \alpha_{1}+a_{011}\right) \alpha_{2}+\left(a_{221} \alpha_{1}^{2}+a_{121} \alpha_{1}+a_{021}\right) \alpha_{2}^{2}\right\} \alpha_{3} \\
& \left.+\left\{a_{202} \alpha_{1}^{2}+a_{102} \alpha_{1}+a_{002}+\left(a_{212} \alpha_{1}^{2}+a_{112} \alpha_{1}+a_{012}\right) \alpha_{2}+\left(a_{222} \alpha_{1}^{2}+a_{122} \alpha_{1}+a_{022}\right) \alpha_{2}^{2}\right\} \alpha_{3}^{2}\right] \\
& +\frac{8 \Delta_{1} \Delta_{2} \Delta_{3}}{3} \times \\
& {\left[\left\{a_{200} \Delta_{1}^{2}+a_{210} \Delta_{1}^{2} \alpha_{2}+a_{220} \Delta_{1}^{2} \alpha_{2}^{2}+a_{020} \Delta_{2}^{2}+a_{120} \alpha_{1} \Delta_{2}^{2}+a_{220} \alpha_{1}^{2} \Delta_{2}^{2}\right\}\right.} \\
& +\left\{a_{201} \Delta_{1}^{2}+a_{211} \Delta_{1}^{2} \alpha_{2}+a_{221} \Delta_{1}^{2} \alpha_{2}^{2}+a_{021} \Delta_{2}^{2}+a_{121} \alpha_{1} \Delta_{2}^{2}+a_{221} \alpha_{1}^{2} \Delta_{2}^{2}\right\} \alpha_{3} \\
& \left.+\left\{a_{202} \Delta_{1}^{2}+a_{212} \Delta_{1}^{2} \alpha_{2}+a_{222} \Delta_{1}^{2} \alpha_{2}^{2}+a_{022} \Delta_{2}^{2}+a_{122} \alpha_{1} \Delta_{2}^{2}+a_{222} \alpha_{1}^{2} \Delta_{2}^{2}\right\} \alpha_{3}^{2}\right] \\
& +\frac{8 \Delta_{1} \Delta_{2} \Delta_{3}}{9}\left(a_{220} \Delta_{1}^{2} \Delta_{2}^{2}+a_{221} \Delta_{1}^{2} \Delta_{2}^{2} \alpha_{3}+a_{222} \Delta_{1}^{2} \Delta_{2}^{2} \alpha_{3}^{2}\right) \\
& +\frac{8 \Delta_{1} \Delta_{2} \Delta_{3}}{3} \times \\
& \left\{a_{202} \alpha_{1}^{2}+a_{102} \alpha_{1}+a_{002}+\left(a_{212} \alpha_{1}^{2}+a_{112} \alpha_{1}+a_{012}\right) \alpha_{2}+\left(a_{222} \alpha_{1}^{2}+a_{122} \alpha_{1}+a_{022}\right) \alpha_{2}^{2}\right\} \Delta_{3}^{2} \\
& +\frac{8 \Delta_{1} \Delta_{2} \Delta_{3}}{9}\left(a_{202} \Delta_{1}^{2}+a_{212} \Delta_{1}^{2} \alpha_{2}+a_{222} \Delta_{1}^{2} \alpha_{2}^{2}+a_{022} \Delta_{2}^{2}+a_{122} \alpha_{1} \Delta_{2}^{2}+a_{222} \alpha_{1}^{2} \Delta_{2}^{2}\right) \Delta_{3}^{2} \\
& +\frac{8 \Delta_{1} \Delta_{2} \Delta_{3}}{27} a_{222} \Delta_{1}^{2} \Delta_{2}^{2} \Delta_{3}^{2}
\end{aligned}
$$


By rearranging terms we have

$$
\begin{aligned}
& \int_{\alpha_{1}-\Delta_{1}}^{\alpha_{1}+\Delta_{1}} \int_{\alpha_{2}-\Delta_{2}}^{\alpha_{2}+\Delta_{2}} \int_{\alpha_{3}-\Delta_{3}}^{\alpha_{3}+\Delta_{3}} w d x d y d z \\
= & 8 \Delta_{1} \Delta_{2} \Delta_{3} \times \\
& {\left[\left\{a_{200} \alpha_{1}^{2}+a_{100} \alpha_{1}+a_{000}+\left(a_{210} \alpha_{1}^{2}+a_{110} \alpha_{1}+a_{010}\right) \alpha_{2}+\left(a_{220} \alpha_{1}^{2}+a_{120} \alpha_{1}+a_{020}\right) \alpha_{2}^{2}\right\}\right.} \\
& +\left\{a_{201} \alpha_{1}^{2}+a_{101} \alpha_{1}+a_{001}+\left(a_{211} \alpha_{1}^{2}+a_{111} \alpha_{1}+a_{011}\right) \alpha_{2}+\left(a_{221} \alpha_{1}^{2}+a_{121} \alpha_{1}+a_{021}\right) \alpha_{2}^{2}\right\} \alpha_{3} \\
& \left.+\left\{a_{202} \alpha_{1}^{2}+a_{102} \alpha_{1}+a_{002}+\left(a_{212} \alpha_{1}^{2}+a_{112} \alpha_{1}+a_{012}\right) \alpha_{2}+\left(a_{222} \alpha_{1}^{2}+a_{122} \alpha_{1}+a_{022}\right) \alpha_{2}^{2}\right\} \alpha_{3}^{2}\right] \\
+ & \frac{8 \Delta_{1} \Delta_{2} \Delta_{3}}{3} \times \\
& {\left[\left\{a_{200}+a_{210} \alpha_{2}+a_{220} \alpha_{2}^{2}+\left(a_{201}+a_{211} \alpha_{2}+a_{221} \alpha_{2}^{2}\right) \alpha_{3}+\left(a_{202}+a_{212} \alpha_{2}+a_{222} \alpha_{2}^{2}\right) \alpha_{3}^{2}\right\} \Delta_{1}^{2}\right.} \\
& +\left\{a_{020}+a_{120} \alpha_{1}+a_{220} \alpha_{1}^{2}+\left(a_{021}+a_{121} \alpha_{1}+a_{221} \alpha_{1}^{2}\right) \alpha_{3}+\left(a_{022}+a_{122} \alpha_{1}+a_{222} \alpha_{1}^{2}\right) \alpha_{3}^{2}\right\} \Delta_{2}^{2} \\
& \left.+\left\{a_{002}+a_{102} \alpha_{1}+a_{202} \alpha_{1}^{2}+\left(a_{012}+a_{112} \alpha_{1}+a_{212} \alpha_{1}^{2}\right) \alpha_{2}+\left(a_{022}+a_{122} \alpha_{1}+a_{222} \alpha_{1}^{2}\right) \alpha_{2}^{2}\right\} \Delta_{3}^{2}\right] \\
+ & \frac{8 \Delta_{1} \Delta_{2} \Delta_{3}}{9} \times \\
& \left\{a_{220} \Delta_{1}^{2} \Delta_{2}^{2}+a_{221} \Delta_{1}^{2} \Delta_{2}^{2} \alpha_{3}+a_{222} \Delta_{1}^{2} \Delta_{2}^{2} \alpha_{3}^{2}+a_{202} \Delta_{1}^{2} \Delta_{3}^{2}+a_{212} \Delta_{1}^{2} \alpha_{2} \Delta_{3}^{2}+a_{222} \Delta_{1}^{2} \alpha_{2}^{2} \Delta_{3}^{2}\right. \\
+ & \left.a_{022} \Delta_{2}^{2} \Delta_{3}^{2}+a_{122} \alpha_{1} \Delta_{2}^{2} \Delta_{3}^{2}+a_{222} \alpha_{1}^{2} \Delta_{2}^{2} \Delta_{3}^{2}\right\} \\
+ & \frac{8 \Delta_{1} \Delta_{2} \Delta_{3}}{27} a_{222} \Delta_{1}^{2} \Delta_{2}^{2} \Delta_{3}^{2} .
\end{aligned}
$$

From (4) we have $27\left(=3^{3}\right)$-data

$$
w_{l, m, n}=w\left(\alpha_{1}+l \Delta_{1}, \alpha_{2}+m \Delta_{2}, \alpha_{3}+n \Delta_{3}\right)
$$

for $l, m, n \in\{-1,0,1\}$, so we must express the integral above in terms of these data $\left\{w_{l, m, n}\right\}$. Since

$$
\begin{aligned}
& \left\{a_{200} \alpha_{1}^{2}+a_{100} \alpha_{1}+a_{000}+\left(a_{210} \alpha_{1}^{2}+a_{110} \alpha_{1}+a_{010}\right) \alpha_{2}+\left(a_{220} \alpha_{1}^{2}+a_{120} \alpha_{1}+a_{020}\right) \alpha_{2}^{2}\right\} \\
+ & \left\{a_{201} \alpha_{1}^{2}+a_{101} \alpha_{1}+a_{001}+\left(a_{211} \alpha_{1}^{2}+a_{111} \alpha_{1}+a_{011}\right) \alpha_{2}+\left(a_{221} \alpha_{1}^{2}+a_{121} \alpha_{1}+a_{021}\right) \alpha_{2}^{2}\right\} \alpha_{3} \\
+ & \left\{a_{202} \alpha_{1}^{2}+a_{102} \alpha_{1}+a_{002}+\left(a_{212} \alpha_{1}^{2}+a_{112} \alpha_{1}+a_{012}\right) \alpha_{2}+\left(a_{222} \alpha_{1}^{2}+a_{122} \alpha_{1}+a_{022}\right) \alpha_{2}^{2}\right\} \alpha_{3}^{2} \\
= & w_{0,0,0}
\end{aligned}
$$

we treat the remaining ones. Here we list formulas necessary to prove the main formula. 


$$
\begin{aligned}
& \left\{a_{200}+a_{210} \alpha_{2}+a_{220} \alpha_{2}^{2}+\left(a_{201}+a_{211} \alpha_{2}+a_{221} \alpha_{2}^{2}\right) \alpha_{3}+\left(a_{202}+a_{212} \alpha_{2}+a_{222} \alpha_{2}^{2}\right) \alpha_{3}^{2}\right\} \Delta_{1}^{2} \\
= & \frac{1}{2}\left(w_{-1,0,0}+w_{1,0,0}\right)-w_{0,0,0}, \\
& \left\{a_{020}+a_{120} \alpha_{1}+a_{220} \alpha_{1}^{2}+\left(a_{021}+a_{121} \alpha_{1}+a_{221} \alpha_{1}^{2}\right) \alpha_{3}+\left(a_{022}+a_{122} \alpha_{1}+a_{222} \alpha_{1}^{2}\right) \alpha_{3}^{2}\right\} \Delta_{2}^{2} \\
= & \frac{1}{2}\left(w_{0,-1,0}+w_{0,1,0}\right)-w_{0,0,0}, \\
& \left\{a_{002}+a_{102} \alpha_{1}+a_{202} \alpha_{1}^{2}+\left(a_{012}+a_{112} \alpha_{1}+a_{212} \alpha_{1}^{2}\right) \alpha_{2}+\left(a_{022}+a_{122} \alpha_{1}+a_{222} \alpha_{1}^{2}\right) \alpha_{2}^{2}\right\} \Delta_{3}^{2} \\
= & \frac{1}{2}\left(w_{0,0,-1}+w_{0,0,1}\right)-w_{0,0,0}
\end{aligned}
$$

and

$$
\begin{aligned}
& \quad a_{220} \Delta_{1}^{2} \Delta_{2}^{2}+a_{221} \Delta_{1}^{2} \Delta_{2}^{2} \alpha_{3}+a_{222} \Delta_{1}^{2} \Delta_{2}^{2} \alpha_{3}^{2}+a_{202} \Delta_{1}^{2} \Delta_{3}^{2}+a_{212} \Delta_{1}^{2} \alpha_{2} \Delta_{3}^{2}+a_{222} \Delta_{1}^{2} \alpha_{2}^{2} \Delta_{3}^{2} \\
& +a_{022} \Delta_{2}^{2} \Delta_{3}^{2}+a_{122} \alpha_{1} \Delta_{2}^{2} \Delta_{3}^{2}+a_{222} \alpha_{1}^{2} \Delta_{2}^{2} \Delta_{3}^{2} \\
& =\frac{1}{4}\left(w_{-1,-1,0}+w_{-1,1,0}+w_{1,-1,0}+w_{1,1,0}+w_{-1,0,-1}+w_{-1,0,1}+w_{1,0,-1}+w_{1,0,1}\right. \\
& \left.\quad \quad+w_{0,-1,-1}+w_{0,-1,1}+w_{0,1,-1}+w_{0,1,1}\right) \\
& \quad-\left(w_{-1,0,0}+w_{1,0,0}+w_{0,-1,0}+w_{0,1,0}+w_{0,0,-1}+w_{0,0,1}\right)+3 w_{0,0,0}
\end{aligned}
$$

and

$$
\begin{aligned}
& a_{222} \Delta_{1}^{2} \Delta_{2}^{2} \Delta_{3}^{2} \\
& =\frac{1}{8}\left(w_{-1,-1,-1}+w_{1,-1,-1}+w_{1,1,-1}+w_{-1,1,-1}+w_{-1,-1,1}+w_{1,-1,1}+w_{1,1,1}+w_{-1,1,1}\right) \\
& -\frac{1}{4}\left(w_{-1,-1,0}+w_{-1,1,0}+w_{1,-1,0}+w_{1,1,0}+w_{-1,0,-1}+w_{-1,0,1}+w_{1,0,-1}+w_{1,0,1}\right. \\
& \left.\quad+w_{0,-1,-1}+w_{0,-1,1}+w_{0,1,-1}+w_{0,1,1}\right) \\
& +\frac{1}{2}\left(w_{-1,0,0}+w_{1,0,0}+w_{0,-1,0}+w_{0,1,0}+w_{0,0,-1}+w_{0,0,1}\right)-w_{0,0,0} .
\end{aligned}
$$

The proofs are long but straightforward, so they are left to readers.

Therefore, substituting (17) $\sim(20)$ into (15) we obtain 


$$
\begin{aligned}
& \int_{\alpha_{1}-\Delta_{1}}^{\alpha_{1}+\Delta_{1}} \int_{\alpha_{2}-\Delta_{2}}^{\alpha_{2}+\Delta_{2}} \int_{\alpha_{3}-\Delta_{3}}^{\alpha_{3}+\Delta_{3}} w d x d y d z \\
& =8 \Delta_{1} \Delta_{2} \Delta_{3} w_{0,0,0} \\
& +\frac{8 \Delta_{1} \Delta_{2} \Delta_{3}}{3}\left\{\frac{1}{2}\left(w_{-1,0,0}+w_{1,0,0}+w_{0,-1,0}+w_{0,1,0}+w_{0,0,-1}+w_{0,0,1}\right)-3 w_{0,0,0}\right\} \\
& +\frac{8 \Delta_{1} \Delta_{2} \Delta_{3}}{9}\left\{\frac { 1 } { 4 } \left(w_{-1,-1,0}+w_{-1,1,0}+w_{1,-1,0}+w_{1,1,0}+w_{-1,0,-1}+w_{-1,0,1}+w_{1,0,-1}+w_{1,0,1}\right.\right. \\
& \left.+w_{0,-1,-1}+w_{0,-1,1}+w_{0,1,-1}+w_{0,1,1}\right) \\
& \left.-\left(w_{-1,0,0}+w_{1,0,0}+w_{0,-1,0}+w_{0,1,0}+w_{0,0,-1}+w_{0,0,1}\right)+3 w_{0,0,0}\right\} \\
& +\frac{8 \Delta_{1} \Delta_{2} \Delta_{3}}{27}\left\{\frac{1}{8}\left(w_{-1,-1,-1}+w_{1,-1,-1}+w_{1,1,-1}+w_{-1,1,-1}+w_{-1,-1,1}+w_{1,-1,1}+w_{1,1,1}+w_{-1,1,1}\right)\right. \\
& -\frac{1}{4}\left(w_{-1,-1,0}+w_{-1,1,0}+w_{1,-1,0}+w_{1,1,0}+w_{-1,0,-1}+w_{-1,0,1}+w_{1,0,-1}+w_{1,0,1}\right. \\
& \left.+w_{0,-1,-1}+w_{0,-1,1}+w_{0,1,-1}+w_{0,1,1}\right) \\
& \left.+\frac{1}{2}\left(w_{-1,0,0}+w_{1,0,0}+w_{0,-1,0}+w_{0,1,0}+w_{0,0,-1}+w_{0,0,1}\right)-w_{0,0,0}\right\} \\
& =\frac{\Delta_{1} \Delta_{2} \Delta_{3}}{27} \times \\
& \left\{w_{-1,-1,-1}+w_{1,-1,-1}+w_{1,1,-1}+w_{-1,1,-1}+w_{-1,-1,1}+w_{1,-1,1}+w_{1,1,1}+w_{-1,1,1}\right. \\
& +4\left(w_{-1,-1,0}+w_{-1,1,0}+w_{1,-1,0}+w_{1,1,0}+w_{-1,0,-1}+w_{-1,0,1}+w_{1,0,-1}+w_{1,0,1}\right. \\
& \left.+w_{0,-1,-1}+w_{0,-1,1}+w_{0,1,-1}+w_{0,1,1}\right) \\
& +16\left(w_{-1,0,0}+w_{1,0,0}+w_{0,-1,0}+w_{0,1,0}+w_{0,0,-1}+w_{0,0,1}\right) \\
& \left.+64 w_{0,0,0}\right\} \text {. }
\end{aligned}
$$

The proof is a bit complicated.

\subsection{General Case}

Let us consider the general case. Unfortunately, we have no method to calculate the general case at the present time. However, from the results (13) and (21) we can conjecture the general formula as follows. 
The formulas (13) and (21) can be rewritten as

$$
\begin{aligned}
& \int_{\alpha_{1}-\Delta_{1}}^{\alpha_{1}+\Delta_{1}} \int_{\alpha_{2}-\Delta_{2}}^{\alpha_{2}+\Delta_{2}} w d x d y \\
= & \frac{\Delta_{1} \Delta_{2}}{3^{2}}\left\{w_{-1,-1}+w_{-1,1}+w_{1,-1}+w_{1,1}+4\left(w_{-1,0}+w_{1,0}+w_{0,-1}+w_{0,1}\right)+4^{2} w_{0,0}\right\}
\end{aligned}
$$

and

$$
\begin{aligned}
& \int_{\alpha_{1}-\Delta_{1}}^{\alpha_{1}+\Delta_{1}} \int_{\alpha_{2}-\Delta_{2}}^{\alpha_{2}+\Delta_{2}} \int_{\alpha_{3}-\Delta_{3}}^{\alpha_{3}+\Delta_{3}} w d x d y d z \\
= & \frac{\Delta_{1} \Delta_{2} \Delta_{3}}{3^{3}} \times \\
& \left\{w_{-1,-1,-1}+w_{1,-1,-1}+w_{1,1,-1}+w_{-1,1,-1}+w_{-1,-1,1}+w_{1,-1,1}+w_{1,1,1}+w_{-1,1,1}\right. \\
& +4\left(w_{-1,-1,0}+w_{-1,1,0}+w_{1,-1,0}+w_{1,1,0}+w_{-1,0,-1}+w_{-1,0,1}+w_{1,0,-1}+w_{1,0,1}\right. \\
& \left.\quad+w_{0,-1,-1}+w_{0,-1,1}+w_{0,1,-1}+w_{0,1,1}\right) \\
& +4^{2}\left(w_{-1,0,0}+w_{1,0,0}+w_{0,-1,0}+w_{0,1,0}+w_{0,0,-1}+w_{0,0,1}\right) \\
& \left.+4^{3} w_{0,0,0}\right\} .
\end{aligned}
$$

From the forms it is easy to conjecture the general form.

If we set

$$
w=F\left(x_{1}, x_{2}, \cdots, x_{n}\right)=\sum_{i_{1}, i_{2}, \cdots, i_{n}=0}^{2} a_{i_{1} i_{2} \cdots i_{n}} x_{1}^{i_{1}} x_{2}^{i_{2}} \cdots x_{n}^{i_{n}}
$$

then

$$
\begin{aligned}
& \int_{\alpha_{1}-\Delta_{1}}^{\alpha_{1}+\Delta_{1}} \int_{\alpha_{2}-\Delta_{2}}^{\alpha_{2}+\Delta_{2}} \cdots \int_{\alpha_{n}-\Delta_{n}}^{\alpha_{n}+\Delta_{n}} w d x_{1} d x_{2} \cdots d x_{n} \\
& =\frac{\Delta_{1} \Delta_{2} \cdots \Delta_{n}}{3^{n}} \sum_{j_{1}, j_{2}, \cdots, j_{n} \in\{-1,0,1\}} 4^{\sharp\left\{j_{1}, j_{2}, \cdots, j_{n}\right\}_{0}} w_{j_{1} j_{2} \cdots j_{n}}
\end{aligned}
$$

where $\sharp\left\{j_{1}, j_{2}, \cdots, j_{n}\right\}_{0}$ is the number of 0 in $\left\{j_{1}, j_{2}, \cdots, j_{n}\right\}$ and

$$
\begin{aligned}
w_{j_{1} j_{2} \cdots j_{n}} & =F\left(\alpha_{1}+j_{1} \Delta_{1}, \alpha_{2}+j_{2} \Delta_{2}, \cdots, \alpha_{n}+j_{n} \Delta_{n}\right) \\
& =f\left(\alpha_{1}+j_{1} \Delta_{1}, \alpha_{2}+j_{2} \Delta_{2}, \cdots, \alpha_{n}+j_{n} \Delta_{n}\right) .
\end{aligned}
$$

The formula is beautiful enough. 


\section{Concluding Remarks}

In the paper we gave a multidimensional analogue of the Simpson's formula of integral. As far as we know the formula has not been given, which is a bit mysterious. We believe that it will become useful in all fields related to integrals.

Next let us consider more general problems. Let $(M, g)$ be a curved space-time and $f$ be a function on $M$. If we consider the integral

$$
\int_{M} f(x) d v_{g}(x)
$$

where $d v_{g}$ is some measure on $M$, it is almost impossible to calculate. For that we want to discretize the integral as follows.

$$
\sum_{\{D\}} \int_{D} f(x) d v_{g}(x) \longrightarrow \sum_{\{D\}}\{\text { some formula like (23) }\}
$$

Therefore we must choose $D$ to be "computable", which is just key point.

Details of calculation will be reported in [2].

\section{References}

[1] WolframMathWorld : http://mathworld.wolfram.com/

[2] K. Fujii : in progress. 